\title{
The Scheme about Biochemical Index Monitoring and Physical Recovery after a Heavy Load Training of Elite Midst \& Long-distance Runners
}

Shenglin Zhang, Xuyang Song, Yang Hao

dept. of PE, Lanzhou University of Technology, Lanzhou, China

ABSTRACT: The purpose of this paper is testing the biochemical indicators changes of elite midst and long distance running athletes after the heave load training, and monitoring functional status and capacity of athletes under the training; this pater takes literature material consult and measurement as research methods; after the heavy load training, the results shows most athletes have the high BUM index and relatively good recovery $\mathrm{CK}$ index, all athletes have normal $\operatorname{IgA}, \operatorname{IgC}$, $\operatorname{IgM}$ index except two; the conclusion is most elite midst and long distance running athletes can adapt the heavy load training quickly, but some athletes are not adapted to the exercise intensity which are reflected in the slow physical recovery and the decreasing of immunologic function. It is important to reinforce the nutritional supplement and adjust exercise intensity for those athletes.

KEYWORDS: Middle distance race; Load; Training; Biochemical indicators; Recovery

In order to preparing the 12th National Games in China, seven male elite midst and long-distance athletes from Gansu Province had the four months long heavy load training at highland. During the training, researchers applied timely monitoring physiological and biochemical indexes for all athletes, which provided useful reference for coaches to make the reasonable exercise intensity arrangements [1]. This paper is about the analysis of biochemical index results and nutritional recovery proposals for those athletes after one heavy load training on highland.

\section{RESEARCH OBJECT AND METHODS}

\subsection{Research object}

This paper takes seven elite midst and long-distance athletes from Gansu province as the research object.

Table 1 shows the information of these athletes.

\begin{tabular}{|c|c|c|c|c|c|c|}
\hline No. & Athletes & Age & Height $(\mathrm{m})$ & Weight $(\mathrm{kg})$ & Training Time(year) & Sports Level \\
\hline 1 & $\mathrm{Mr} . \mathrm{Li}$ & 25 & 1.75 & 58 & 11 & international athlete \\
\hline 2 & $\mathrm{Mr} . \mathrm{Chen}$ & 24 & 1.76 & 60 & 4 & national 1st level \\
\hline 3 & $\mathrm{Mr} . \mathrm{Hu}$ & 23 & 1.78 & 61 & 4 & national 1st level \\
\hline 4 & $\mathrm{Mr} . \mathrm{Qu}$ & 22 & 1.77 & 59 & 7 & sportsperson \\
\hline 5 & $\mathrm{Mr} . \mathrm{Sun}$ & 21 & 1.76 & 60 & 7 & sportsperson \\
\hline 6 & Mr. Wang & 25 & 1.78 & 61 & 7 & sportsperson \\
\hline 7 & Mr. Chen & 24 & 1.77 & 62 & 7 & sportsperson \\
\hline
\end{tabular}

\subsection{Research Methods}

\subsubsection{Literature Material Consult:}

Consulted a number literature material which are related to biochemical index testing of midst and long-distance running training.

\subsubsection{Measurement:}

Applied tests for all athletes about blood lactic acid 
index, serum CK index, serum testosterone (T), cortisol index after different stage of training.

\section{RESULTS AND ANALYSIS}

2.1 The biochemical index test results of seven elite male midst and long-distance runners after the heavy load training.

Table 2 The Biochemical Index Test results of Seven Elite Male Midst and Long-distance Runners After the Heavy Load Training

\begin{tabular}{|c|c|c|c|c|c|c|c|c|c|c|}
\hline Name & Sex & HGB $(\mathrm{g} / \mathrm{L})$ & $\mathrm{T}(\mathrm{ng} / \mathrm{dl})$ & $\mathrm{T} / \mathrm{C}$ & $\mathrm{BUN}(\mathrm{mmol} / \mathrm{L})$ & $\mathrm{CK}(\mathrm{U} / \mathrm{L})$ & BLU & $\operatorname{IgA}(\mathrm{g} / \mathrm{L})$ & $\operatorname{IgG}(\mathrm{g} / \mathrm{L})$ & $\operatorname{IgM}(\mathrm{g} / \mathrm{L})$ \\
\hline $\mathrm{Mr}$. Li & male & 14.5 & 460 & 0.02 & 7.9 & 390 & 4 & 2.1 & 16.5 & 0.4 \\
\hline Mr.Chen & male & 16.5 & 580 & 0.05 & 8 & 410 & 4.2 & 1.82 & 9.5 & 1.4 \\
\hline $\mathrm{Mr}$. Hu & male & 15 & 490 & 0.08 & 7.8 & 350 & 4.1 & 1.4 & 10 & 0.5 \\
\hline $\mathrm{Mr}$ Qu & male & 15.6 & 540 & 0.02 & 7.6 & 290 & 3.95 & 1.91 & 13 & 0.46 \\
\hline Mr. Sun & male & 15.1 & 470 & 0.02 & 8.4 & 180 & 3.2 & 1.9 & 12.5 & 0.45 \\
\hline Mr.Wang & male & 16.8 & 590 & 0.02 & 7.9 & 392 & 4.15 & 2.2 & 17.2 & 1.35 \\
\hline Mr. Chen & male & 15.9 & 510 & 0.02 & 7.9 & 200 & 3.7 & 1.6 & 5.5 & 0.5 \\
\hline
\end{tabular}

\subsection{Results Analysis and Nutrition Plan}

\subsubsection{The Overall Test Results Analysis and Nutrition Plan for Seven Athletes}

The test results in table 1 shows that all athletes have high urea nitrogen index(reference value 1.7-7.0 $\mathrm{mmol} / \mathrm{L}$ ), main reasons to lead this results are:1)Athletes did large amount of exercise before tests;2)carbohydrate supplement shortage, which increased the proportion of protein metabolism; 3)compare with previous training, the catabolism has intensified during the training on the highland area.

Except some athletes (Mr.Li, Mr.Chen, Mr.Wang) have relatively high CK index, the CK index recovery of other athletes is relatively good (reference value $24-195 \mathrm{U} / \mathrm{L})^{[2]}$. The training should take more consideration about muscle protection and reduce muscle membrane damage.

The glucose index of some athletes (Mr.Sun, Mr.Chen) is below the normal range (reference value 3.9-6.1 mmol/L), have enough supplement of sports drinks and staple foods.

Some athletes (Mr.Li, Mr.Wang) may have injury or infection phenomenon, because their IgA index (reference value $0.5-1.9 \mathrm{~g} / \mathrm{L}$ ) ${ }^{[2]}$ and $\mathrm{IgG}$ index (reference value $8-16 \mathrm{~g} / \mathrm{L}$ ) are above the normal range; it is common that athletes have low IgM index value (reference value 1.4-4.2 $\mathrm{g} / \mathrm{L}$ ), because IgM is immunoglobulin at the early stage. So there is some potential factors to decrease athletes' immune function, it is important to improve athletes' immunity at early stage of training.

The morning urine protein of some athletes (Mr.Li) shows the positive phenomenon (reference value $105-175 \mathrm{~g} / \mathrm{L})^{[2]}$. The great training intensity may relate to this phenomenon, but this athlete didn't have occult blood. Further tracking observe is necessary to find out if this athlete had exercise fatigue.
The overall analysis shows that after the heavy load training some athletes didn't adapt the exercise strength, which leads to the poor regeneration and decreased immune function. The coach should take actions to improve athletes' immunity at the early stage of training.

For some common problems [3], such as high urea nitrogen index, and hypoglycemia. Because glycogen shortage strengthen the protein supplement, and increased the metabolite. More staple food should be added to the meal for athletes, and provide sports drinks supplement before, during, and after the training. For example, the sugar supplement amount should be 1.2-1.4 g/kg; a reasonable amount of albumen power $(0.3-0.5 \mathrm{~g} / \mathrm{kg})$ is good for the glycogen recovery after the heavy load training ${ }^{[4]}$; athletes should have the supplement of amino acid, such as glutamine, which is close related to the immunity. More milk, mushroom, and cooked tomato should add to the meal for athletes.

\subsubsection{Results Analysis and Nutrition Plan for Individual Athlete (random drawing two athletes form the group as the example)}

\subsubsection{Mr. Li}

The test results from table 2 shows that Mr. Li didn't adapt the early stage of training very well, and he should pay more attention on the recovery after training. Suggestions:1)have the supplement of iron element to keep and increase hemoglobin index; 2)increase the testosterone index to increase the ability of composition and recovery; 3)keep increase the supplement proportion of carbohydrate and staple food before, during, after the training; 4)improve immunity and protect athlete from getting cold before the National Games. 
Table 3 The Nurtrition Recovery Plan for Mr. Li

\begin{tabular}{|l|l|}
\hline Time & Plan \\
\hline before breafast & 2 bottles of Tingwei oral liquid, 2 bottles of Changbai Jingxianling oral liquid \\
\hline after breafase & 1 piece of Acclerate Hematopoiesis Centrum \\
\hline before training & 4 granules of Active Capbohydrate Capsule \\
\hline during training & $\begin{array}{l}\text { Comepelex Energy Drink, 2 Granules of Salt Stick ( accorting to the sweat situation may increase or } \\
\text { decrease) }\end{array}$ \\
\hline after training & 5 gram of CPT, 1 spoon of Soybean Peptides Powder, 4 granules of Glutamine Capsules \\
\hline before sleep & $\begin{array}{l}2 \text { bottles of Tingwei oral liquid, 2 bottles of Changbai Jingxianling oral liquid, 2-4 granules of Motor nerve } \\
\text { element, (to adjust neurological function before games or during the heavy load training) }\end{array}$ \\
\hline
\end{tabular}

\subsubsection{2 $\mathrm{Mr} . \mathrm{Hu}$}

The test results form table 2 shows that $\mathrm{Mr}$. $\mathrm{Hu}$ has pretty good overall functional situation, here are some suggestions while keep the same exercise strength: 1) keep increase the supplement proportion of carbohydrate and staple food before, during, after the training; 2) keep and increase hemoglobin index;3) keep and increase the testosterone index; 4) improve immunity and avoid immune function decline while increase the exercise strength.

Table 4 The Nutrition Recovery Plan for Mr. $\mathrm{Hu}$

\begin{tabular}{|c|c|}
\hline Time & Plan \\
\hline before breakfast & 2 bottles of Tingwei oral liquid, 2 bottles of Changbai Jingxianling oral liquid \\
\hline after breakfast & 1 piece of Acclerate Hematopoiesis Centrum \\
\hline before training & 4 granules of Active Capbohydrate Capsule \\
\hline during training & $\begin{array}{l}\text { Comepelex Energy Drink, } 2 \text { Granules of Salt Stick ( accorting to the sweat situation may increase or } \\
\text { decrease) }\end{array}$ \\
\hline after training & 4 granules of polypeptide 1-6 Fructose-1, 6-Diphosphate Sodium per day Glutamine Capsules \\
\hline before sleep & $\begin{array}{l}2 \text { bottles of Tingwei oral liquid, } 2 \text { bottles of Changbai Jingxianling oral liquid, } 2-4 \text { granules of Motor } \\
\text { nerve element (to adjust neurological function before games or during the heavy load training) }\end{array}$ \\
\hline
\end{tabular}

\section{CONCLUSION AND SUGGESTIONS}

\subsection{Conclusion}

(1)After the heavy load training, athletes have the high urea nitrogen index and relatively good CK recovery. The blood glucose index, IgA, IgA, IgA are normal except two athletes.

(2)Athletes from the sub-plateau area may have quick reaction about the training on the highland area.

\subsection{Suggestions}

(1)Increase the test time about pulse in the morning, and the test about heart rate recovery 3 minutes after the training to get the recovery information about athletes;

(2)Strengthen the training about speed endurance, and keep lactic acid index at the relatively stable level under the same exercise strength;

(3)While increase the exercise strength and speed endurance training, it is important to keep good nutrition plan and add some physical relaxing method for athletes to ensure the training for next day;

\section{REFERENCES}

[1] Madongliang, Zhangshenglin. Biochemical Index Monitoring of Marathon Runner Lizhuhong during the training at the highland area. Journal of Physical Education, 2009.6:98-101

[2] Fenglianshi, Likaigong. Common Test Methods and Application about Evaluate Athletes' Biochemical Index. Beijing: Peoples Sports Publishing House, 2002.8: 25-75

[3] Wangqing, Fenglianshi, Wengqingzhang. Training at the Highland Area, Beijing: Peoples Sports Publishing House, 2007.4:88-122

[4] Chenguang, Zhoutiemin, Xiongxibei. The Analysis about Training Strength of Chinese Elite Marathon Runner Hujungang. Chinese Sports Technology, 2002.(38)10:1519

[5] Wangqing. The Research and Establish about Diagnosing and Monitoring Chinese Elite Athletes' Competitive ability and condition. Beijing: Peoples Sports Publishing House, 2004.2:5-10 